\title{
Antibacterial Effects of Uvilla (Physalis peruviana L.) extracts against Listeria spp. Isolated from Meat in Ecuador
}

\author{
Ma. Bernarda Ruilova Cueva1, Rosa Angelica Tigre León', Ma. Magdalena López , \\ Angel Yanchaliquín ${ }^{1}$, Isidro Favian Bayas Morejón ${ }^{1 *}$ and Herminia Sanaguano Salguero² \\ ${ }^{1}$ Centro de Investigación y Desarrollo Biotecnológico, Departamento de Investigación, \\ Universidad Estatal de Bolívar, EC-020150, Guaranda - Ecuador \\ ${ }^{2}$ Centro de Investigación Ambiental, Departamento de Investigación, Universidad Estatal de \\ Bolívar, EC-020150, Guaranda-Ecuador \\ *Corresponding author
}

\author{
A B S T R A C T
}

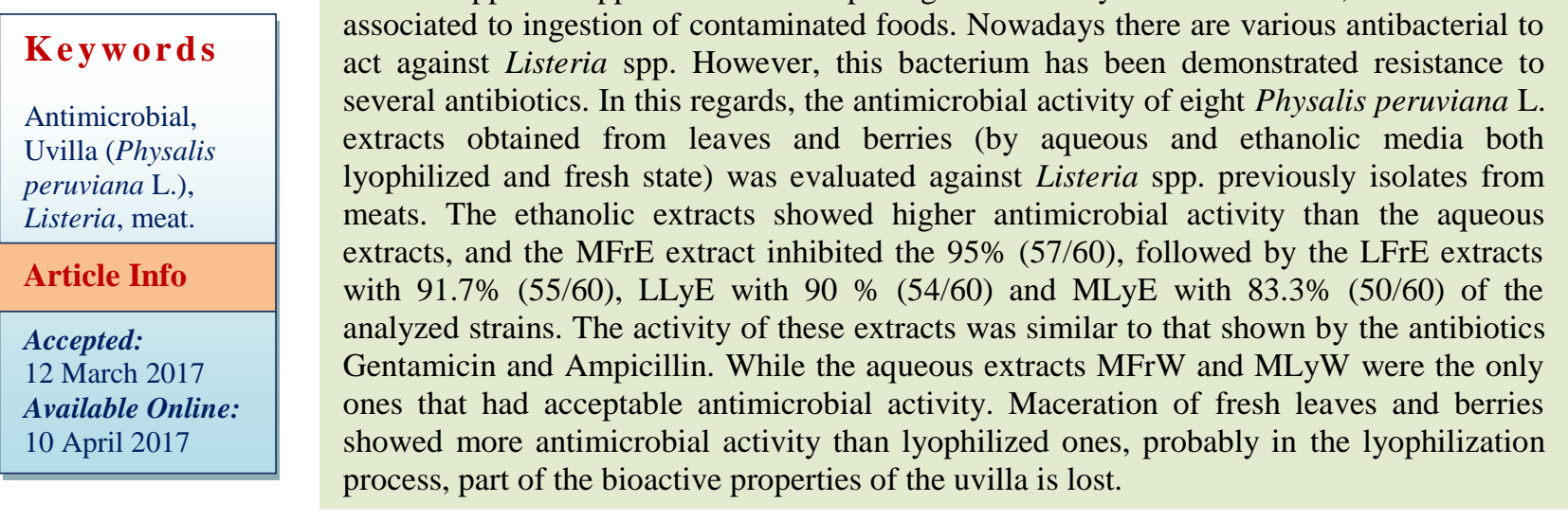

\section{Introduction}

An antibacterial agent is a substance that kills or inhibits the growth of bacteria. Antibacterial substance can be of two types bactericidal and bacteriostatic (Huang et al., 2016). Antibiotic resistance is currently the greatest challenge to effective treatment of infections globally. The continuous emergence of new antibiotic resistant strains day by day has become a major problem for patients (Bayas Morejón et al., 2017; Chavan and Bansode, 2015; Thu Vu et al., 2016). Bacteria have the genetic ability to transfer and acquire resistance to drugs, which are utilized as therapeutic agents (Khushwaha et al., 2012). Therefore, alternatives to these chemical antibiotics have become necessary. The side effect associated with the available antibiotics is alarming too. Hence, with the increase of microbial resistance to antibiotics, there is considerable interest in investigating the antimicrobial effects of different types of plant extracts as potential sources of natural antimicrobials against a wide range of microorganisms. 
Medicinal plants are important therapeutic agents for medicine due to their alternatively to chemical products and most of plants have valuable bioactive phytochemicals compounds. Besides plants, fruits also have been studied by the researchers for the presence of bioactive compounds close related to herbs, commonly referred as phytochemicals such as tannins, carotenoids, polyphenols and anthocyanins (Khushwaha et al., 2012). Crude extracts from plants with a history of use in folk medicines have been screened in vitro for antibacterial activity by many research groups (Suzgec-Selcuk and Birteksoz, 2011).

Physalis peruviana L. (golden berry) is a member of solanaceae family. The fruits of golden berry have high nutritional value because of possessing high minerals, antioxidants and vitamins content (Cakir et al., 2014).

These plants have also potential medicinal properties like anti-bacterial, antiinflammatory, and antioxidant properties (Corrales-Bernal et al., 2015; Göztok and Zengin, 2013; Yen, et al., 2010). Besides this plant is known to induce apoptosis in different phases and has anti-cancer activity (Yen et $a l ., 2010)$. Therefore, the interest in this genus has grown in many regions of the world in recent decade.

Listeria is a widely distributed bacterium in nature and commonly found in soil, sewage, dust, water and causes listeriosis in humans and animals. Listeriosis is a relatively rare food-borne illness, but can be life threatening with high fatality rates. It is mainly associated with the consumption of processed foods that require no further cooking by the consumer (Shantha and Gopal, 2014).

In Ecuador, listerisis have been associated with foods especially of animal origin and water (DNVE-Ecuador, 2016). During 2013, in Ecuador there were 163 deaths from intestinal infectious diseases, according to the Ministry of Health. Of these, 141 were due to diarrhea and gastroenteritis of presumed infectious origin (MSP, 2014).

Is of our known that there are not data about of the antilisterial activity of Physalis peruviana L. The sensitivity of amicroorganism towards an antimicrobial agent can be tested using the antimicrobial susceptibility test. Conventionally, antimicrobial test results are reported qualitatively and/or quantitatively. Qualitative results are often reported as susceptible $(\mathrm{S})$, intermediate (I), or resistant (R) (Coyle, 2005).

Our objective was to study the antilisterial activity of Physalis peruvina L. extract on fruits and leaves against Listeria spp. strains isolated from meat.

\section{Materials and Methods}

\section{Listeria spp. strains}

Sixty strains of Listeria spp. were used in this study. All strains, belonging to the Department of Investigation, Centro de Investigación y Desarrollo Biotecnógico de la Universidad Estatal de Bolívar (Ecuador), were previously isolated from three types of meats (beef, pork and chicken), following the ISO method 11290e1:1996 (ISO 11290e1:1996). Bacterial strains were stored in cryovials (Microbank $^{\mathrm{TM}}$ Prolab Diagnostics, USA) at $-80{ }^{\circ} \mathrm{C}$.

The strains were reactivated and sub-cultured onto Chromogenic Listeria Agar (CLA, 7502A, Neogen, USA), incubated overnight at $37{ }^{\circ} \mathrm{C}$. The reference strain (Listeria innocua "ATCC 33090") was rehydrated and cultured according to the Culture Collections instructions. 


\section{Uvilla samples and extraction of extracts}

The extracts analyzed in this study were obtained from the mature berries and leaves of Physalis peruviana L. purchased of different local retail shops from the city of Guaranda (Ecuador) and collected during May 2016 to January 2017.

The berries and healthy leaves were washed and triturated, in parallel also were lyophilized in a Freeze drier (Labconco FreeZone 2.5, USA), for their later processed.

Five, teen and five teen grams of uvilla (berries and leaves) were put to maceration in $100 \mathrm{~mL}$ of $95 \%$ ethanol and $100 \mathrm{~mL}$ of distiller water at room temperature for $6 \mathrm{~d}$. according to the method established by Cakir et al. (2014) and Areiza et al. (2013) with some modifications. In table 1, the treatments performed in this research are detailed.

The extract was centrifuged while the residue was further extracted under the same conditions twice. The supernatant collected from separate extractions and were stored at 4 ${ }^{\circ} \mathrm{C}$ in flasks amber.

\section{Screening of antilisterial activity}

The antibacterial activity of the eight extracts against Listeria spp. strains was tested by the paper disc diffusion method applied by Shokeen et al. (2009).

Colonies of fresh pure culture from each isolate and of the references strains (Listeria innocua Seeliger ATCC 33090) were suspended in Saline water 5\%, until the turbidity was adjusted to match the McFarland 0.5 standards. Bacteria from each suspension were inoculated onto Muller Hilton Agar (7101A, Neogen, MichiganUSA) using a sterile cotton-tipped swab and the plates were left standing for a few minutes.
Sterile filter paper discs (TSMX 7215, Oxoid, UK) of $6 \mathrm{~mm}$ diameter were applied to the agar surface and soaked with $10 \mathrm{~mL}$ of each extract (Randazzo et al., 2016). Sterile water was used as negative control. The commercially available standard antibiotics, gentamicin and ampicillin (CN C1333; AN C133, Bioanalyse, Turkey) were used as reference antibiotic controls. All assays were performed in duplicate.

\section{Statistical analysis}

Statistical analyses of the data were undertaken using Statistix IX (Tallahassee University, Florida, USA). The results were subjected to statistical analysis; differences between the eight treatments (extracts) were carried out by Tukey's test. A probability value of less than $5 \%$ was deemed to be significant.

\section{Results and Discussion}

\section{Screening of antibacterial activity}

The antibacterial activity of Physalis peruviana L. extract of both solvents (ethanolic and aqueous) against Listeria isolates by the disk diffusion method are shown in table 2. Most of the extracts resulted statistically different $(\mathrm{P} \leq 0.001$ and $\mathrm{P} \leq 0.01)$ in inhibiting the strains studied, with a range of 2-8 mm. According to Settani et al. (2014), the sensitivity to natural agents depends on the type of isolate, this explains the existing variability.

In this study extracts MFrE, LFrE, LlyE and MLyE showed antibacterial activity against the majority of studied strains. As shown in figure 1, ethanolic extracts showed considerably more activity than the aqueous extract. Nair et al. (2005) demonstrated that alcoholic plant extracts inhibit a greater number of pathogens than aqueous extracts. 
This is because alcohol retains the bioactive components of plants better (Yilmaztekin et al., 2014).

In fact the MFrE extract showed antimicrobial activity in 95\% (57/60) of the Listeria strains analyzed, only strains Lspp 7, Lspp 24 and Lspp 47were not inhibited for this extract. Other extracts with a high number of susceptible strains were LFrE with $91.7 \%$ (55/60), LLyE with 90\% (54/60) and MLyE with $83.3 \%$ (50/60).

Of the aqueous extracts, only MFrW extract had a high antibacterial effect with $73.3 \%$ (44/60) of the analyzed strains, whereas in the other aqueous extracts, the inhibition was in less than $50 \%$ of the strains.

In studies developed by Göztok and Zengin, (2013) and Çakir et al. (2014), evaluated the antimicrobial activity of Physalis peruviana $\mathrm{L}$ versus Bacillus megaterium DSM 32, Proteus vulgaris FMC 1, Klebsiella pneumoniae FMC 66032, Escherichia coli ATCC 25922, Pseudomonas aeruginosa DMS 50071, Enterobacter aeregenes CCM 2531, Staphylococcus aureus A950277, Staphylococcus epidermidis 14990, Lactococcus lactis ATCC 11454, Escherichia coli DH5- $\alpha$ and Erwinia herbicola, But the authors did not evaluate the activity of the extracts against Listeria spp. The extracts from berries and leaves lyophilized had a lower antimicrobial effect than those extracts from fresh state berries and leaves (Figure 1). A disadvantage of the lyophilizate is that apart from being expensive, in the freezedrying process some of the bioactive properties of the plants are lost (Otobone et al., 2007). The reference strain L. innocua (ATCC 33090) presented the same activity to the obtained extracts as the strains tested.

It is the first time that the antimicrobial effect of Physalis peruviana L. extracts against Listeria spp. has been studied. Is also the first time that the difference between leaves and berries lyophilized versus non-lyophilized berries and leaves has been studied.

According to studies of characterization of the uvilla, the phenolic components are the most abundant in both leaves and berries, the most outstanding being 1-hexanol, eucalyptol and 4-terpenol (Yilmaztekin et al., 2014; Corrales-Bernal et al., 2015), as well as organic acids with antioxidant properties (Ramadan et al., 2015). There are studies that have demonstrated that monoterpenes are the components that act in the inhibition of microorganisms (Ramadan et al., 2015, Randazzo et al., 2015).

Table.1 Plant's parts and treatments of maceration for obtaining extracts

\begin{tabular}{lll}
\hline Extracts & Plant's parts & Treatments \\
\hline MFrE & Mature berries & 15 gr in fresh state, maceration in ethanol \\
MFrW & Mature berries & 15 gr in fresh state, maceration in distilled water \\
MLyE & Mature berries & 5 gr lyophilized, maceration in ethanol \\
MLyW & Mature berries & 5 gr lyophilized, maceration in distilled water \\
LFrE & Leaves & 10 gr in fresh state, maceration in ethanol \\
LFrW & Leaves & 10 gr in fresh state, maceration in distilled water \\
LLyE & Leaves & 5 gr lyophilized, maceration in ethanol \\
LLyW & Leaves & 5 gr lyophilized, maceration in distilled water \\
\hline
\end{tabular}


Table.2 Inhibitory activity of Physalis peruviana L. extracts against Listeria spp. isolated from meat tested by disc diffusion assay

\section{Extracts}

\begin{tabular}{|c|c|c|c|c|c|c|c|c|c|c|c|c|}
\hline $\begin{array}{l}\text { Strain } \\
\text { code }\end{array}$ & MFrE & MFrW & MLyE & MLyW & LFrE & LFrW & LLyE & LLyW & $\begin{array}{c}\text { Statistical } \\
\text { significance }\end{array}$ & $\mathrm{AM}$ & $\mathrm{CN}$ & Source \\
\hline Lspp.1 & 2 & 2 & 2 & 2 & 6 & 0 & 3 & 0 & $*$ & 2 & 2 & pork \\
\hline Lspp. 2 & 2 & 0 & 3 & 2 & 1 & 0 & 5 & 0 & $*$ & 2 & 3 & pork \\
\hline Lspp.3 & 3 & 2 & 2 & 1 & 8 & 0 & 2 & 0 & $* *$ & 1 & 3 & chicken \\
\hline Lspp.4 & 2 & 0 & 4 & 3 & 2 & 0 & 4 & 0 & $* *$ & 0 & 1 & chcken \\
\hline Lspp.5 & 1 & 0 & 5 & 1 & 2 & 0 & 2 & 0 & $* * *$ & 2 & 2 & pork \\
\hline Lspp.6 & 2 & 1 & 3 & 2 & 2 & 0 & 2 & 0 & $*$ & 0 & 1 & chicken \\
\hline Lspp.7 & 0 & 0 & 0 & 1 & 5 & 0 & 5 & 0 & $* * *$ & 0 & 2 & chicken \\
\hline Lspp. 8 & 2 & 0 & 7 & 0 & 0 & 0 & 0 & 0 & $* * *$ & 5 & 2 & chicken \\
\hline Lspp.9 & 4 & 0 & 4 & 0 & 0 & 0 & 0 & 0 & $* * *$ & 0 & 1 & chicken \\
\hline Lspp.10 & 2 & 0 & 7 & 0 & 3 & 0 & 4 & 0 & $* * *$ & 5 & 4 & chicken \\
\hline Lspp.11 & 4 & 0 & 1 & 0 & 0 & 0 & 1 & 0 & $* *$ & 5 & 5 & pork \\
\hline Lspp.12 & 5 & 2 & 0 & 0 & 2 & 0 & 2 & 0 & $* *$ & 3 & 6 & beef \\
\hline Lspp.13 & 3 & 5 & 0 & 0 & 0 & 0 & 0 & 0 & $* * *$ & 3 & 7 & beef \\
\hline Lspp.14 & 1 & 2 & 0 & 0 & 0 & 0 & 0 & 0 & Ns & 2 & 3 & beef \\
\hline Lspp.15 & 2 & 2 & 4 & 0 & 1 & 0 & 3 & 0 & $* *$ & 9 & 9 & pork \\
\hline Lspp.16 & 2 & 2 & 3 & 1 & 3 & 0 & 2 & 0 & $*$ & 1 & 9 & chicken \\
\hline Lspp.17 & 3 & 1 & 2 & 0 & 5 & 0 & 4 & 0 & $* * *$ & 1 & 9 & chicken \\
\hline Lspp.18 & 5 & 2 & 2 & 0 & 4 & 0 & 4 & 0 & $* * *$ & 5 & 12 & pork \\
\hline Lspp.19 & 2 & 0 & 1 & 0 & 4 & 0 & 2 & 0 & $* * *$ & 3 & 10 & pork \\
\hline Lspp.20 & 2 & 1 & 1 & 0 & 4 & 0 & 3 & 0 & $* *$ & 10 & 10 & beef \\
\hline Lspp.21 & 3 & 2 & 3 & 0 & 2 & 0 & 2 & 0 & $* *$ & 12 & 12 & beef \\
\hline Lspp.22 & 2 & 0 & 2 & 0 & 4 & 0 & 2 & 0 & $* *$ & 14 & 14 & beef \\
\hline Lspp. 23 & 1 & 1 & 2 & 0 & 4 & 0 & 3 & 0 & $* *$ & 1 & 1 & pork \\
\hline Lspp.24 & 0 & 0 & 0 & 7 & 2 & 0 & 2 & 0 & $* * *$ & 1 & 2 & chicken \\
\hline Lspp. 25 & 5 & 0 & 2 & 0 & 4 & 0 & 3 & 0 & $* *$ & 2 & 10 & pork \\
\hline Lspp.26 & 4 & 1 & 1 & 0 & 4 & 0 & 7 & 0 & $* * *$ & 15 & 15 & pork \\
\hline Lspp. 27 & 2 & 3 & 3 & 0 & 3 & 0 & 6 & 0 & $* * *$ & 7 & 12 & pork \\
\hline Lspp. 28 & 5 & 2 & 5 & 0 & 2 & 0 & 6 & 0 & $* * *$ & 15 & 15 & beef \\
\hline Lspp.29 & 3 & 3 & 2 & 0 & 2 & 0 & 4 & 0 & $* *$ & 12 & 14,5 & beef \\
\hline Lspp.30 & 2 & 2 & 2 & 0 & 2 & 0 & 3 & 0 & $*$ & 12 & 14 & beef \\
\hline Lspp.31 & 2 & 2 & 3 & 3 & 5 & 0 & 3 & 0 & $* *$ & 5 & 1 & chicken \\
\hline Lspp.32 & 3 & 3 & 2 & 3 & 4 & 0,5 & 4 & 0 & $*$ & 5 & 2 & chicken \\
\hline Lspp.33 & 2 & 1 & 3 & 0 & 3 & 0 & 2 & 0 & $* *$ & 2 & 2 & pork \\
\hline Lspp.34 & 4 & 2 & 5 & 0 & 4 & 0 & 2 & 0 & $* * *$ & 2 & 2 & pork \\
\hline Lspp.35 & 3 & 3 & 2 & 0,5 & 3 & 0 & 3 & 0 & $* *$ & 3 & 3 & beef \\
\hline Lspp.36 & 2 & 0 & 3 & 0 & 2 & 0 & 2 & 0 & $* * *$ & 7 & 12 & chicken \\
\hline Lspp.37 & 4 & 2 & 5 & 0 & 4 & 0 & 3 & 0 & $* * *$ & 5 & 2 & beef \\
\hline Lspp.38 & 2 & 1 & 0 & 3 & 2 & 0 & 3 & 0 & $* * *$ & 0 & 2 & chicken \\
\hline Lspp.39 & 1 & 2 & 1 & 1 & 2 & 0 & 1 & 0 & Ns & 0 & 1 & chicken \\
\hline Lspp.40 & 3 & 2 & 3 & 0 & 3 & 0 & 4 & 0 & $* *$ & 10 & 15 & beef \\
\hline
\end{tabular}




\begin{tabular}{lcccccccccccc}
\hline Lspp.41 & 2 & 3 & 2 & 1 & 2 & 0 & 0 & 0 & $* * *$ & 3 & 10 & chicken \\
Lspp.42 & 2 & 3 & 1 & 1 & 2 & 0 & 0 & 0 & $* *$ & 3 & 9 & chicken \\
Lspp.43 & 2 & 3 & 0,5 & 0 & 2 & 0 & 2 & 0 & $* *$ & 4 & 10 & chicken \\
Lspp.44 & 2 & 2 & 6 & 0 & 3 & 0 & 3 & 0 & $* * *$ & 5 & 13 & chicken \\
Lspp.45 & 2 & 3 & 0 & 0 & 2 & 0 & 2 & 0 & $* * *$ & 3 & 10 & pork \\
Lspp.46 & 1 & 0 & 0 & 0 & 4 & 0 & 4 & 0 & $* * *$ & 0 & 4 & pork \\
Lspp.47 & 0 & 0 & 2 & 0 & 3 & 0 & 3 & 0 & $* * *$ & 0 & 0 & pork \\
Lspp.48 & 1 & 1 & 1 & 0 & 5 & 0 & 4 & 0 & $* * *$ & 0 & 0 & beef \\
Lspp.49 & 3 & 2 & 0 & 1 & 1 & 0 & 1 & 0 & $* *$ & 0 & 8 & chicken \\
Lspp.50 & 2 & 0 & 1 & 0 & 1 & 0 & 2 & 0 & Ns & 2 & 2 & chicken \\
Lspp.51 & 4 & 1 & 2 & 0 & 3 & 0 & 5 & 0 & $* * *$ & 5 & 10 & chicken \\
Lspp.52 & 1 & 1 & 2 & 0 & 3 & 0 & 3 & 0 & $* *$ & 5 & 2 & pork \\
Lspp.53 & 4 & 3 & 3 & 1 & 4 & 0 & 4 & 0 & $* *$ & 2 & 1 & pork \\
Lspp.54 & 1 & 3 & 0 & 1 & 2 & 0 & 2 & 0 & $*$ & 1 & 1 & pork \\
Lspp.55 & 2,5 & 1 & 0,5 & 1 & 5 & 0 & 3 & 0 & $* * *$ & 5 & 2 & chicken \\
Lspp.56 & 4 & 3 & 1 & 2 & 2 & 0 & 3 & 0 & $* *$ & 5 & 8 & chicken \\
Lspp.57 & 5 & 1 & 4 & 1 & 2 & 0 & 8 & 1 & $* * *$ & 9 & 10 & chicken \\
Lspp.58 & 1 & 1 & 2 & 1 & 3 & 0 & 4 & 0 & $* * *$ & 5 & 1 & chicken \\
Lspp.59 & 1 & 1 & 1 & 1 & 3 & 0 & 2 & 0 & $* *$ & 0 & 0 & chicken \\
Lspp.60 & 2 & 1 & 3 & 1 & 1 & 0 & 3 & 0 & $* *$ & 5 & 1 & chicken \\
ATCC & 3 & 2 & 3 & 1 & 2 & 0 & 3 & 0 & $* * *$ & 5 & 4 & \\
33090 & & & & & & & & & & & &
\end{tabular}

$\mathrm{P}$ value: *, $\mathrm{P} \leq 0.05 ;{ }^{* *}, \mathrm{P} \leq 0.01 ;{ }^{* *}, \mathrm{P} \geq 0.001 ; \mathrm{ns}$, not significant. The values are expressed in $\mathrm{mm}$.

Fig.1 Number of inhibited strains according to the analyzed extracts

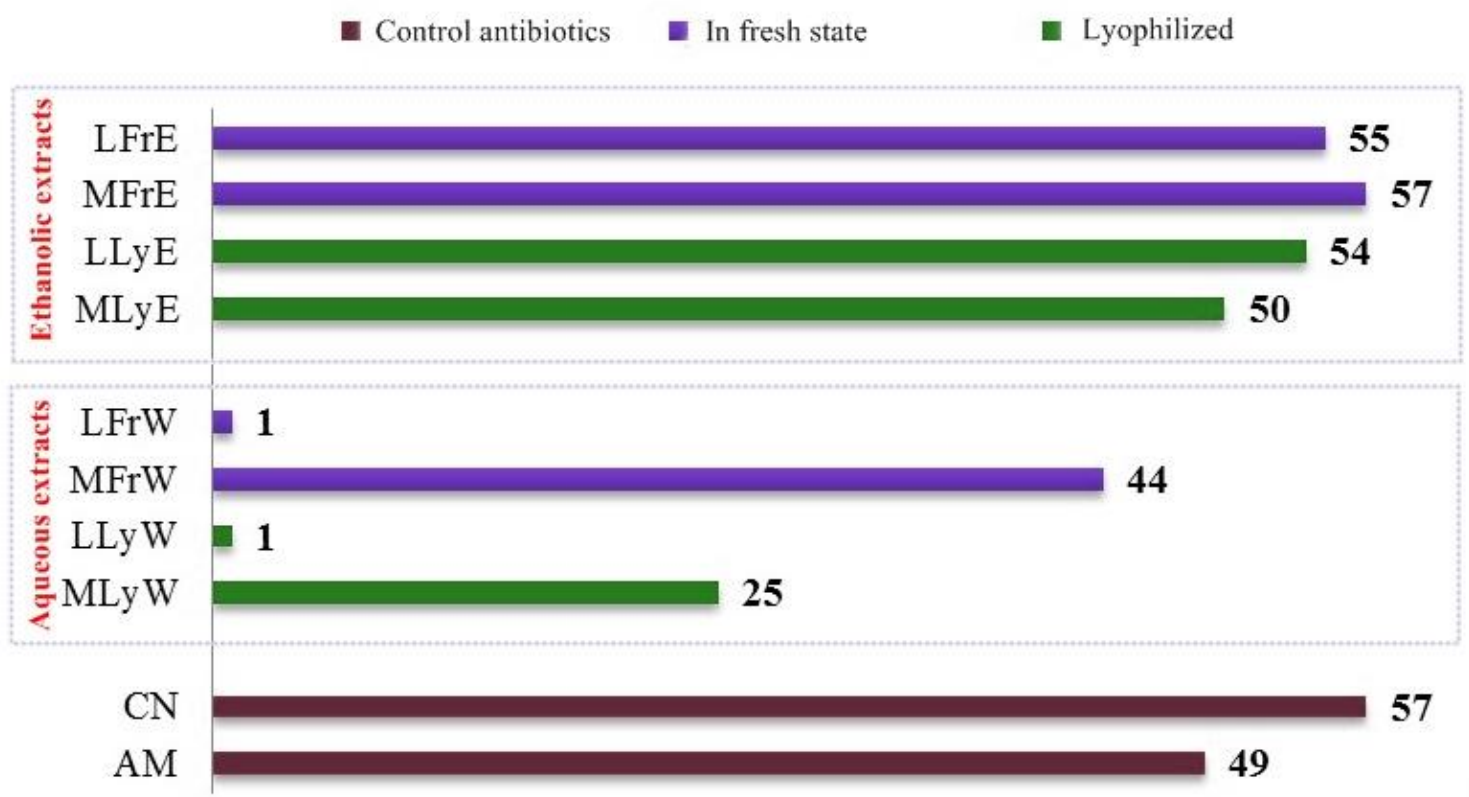

In addition, all Listeria spp. Strains were studied for their susceptibility to Gentamicin (CN) and Ampicillin (AM). CN was effective against $95 \%$ of the analyzed strains, whereas AM acted against $81.7 \%$ of strains. 
On the other hand, the isolated Lspp. 47, Lspp. 48 and Lspp. 59 showed resistance to the two control antibiotics studied, whereas the strains Lspp.4, Lspp. 6, Lspp. 7, Lspp. 9, Lspp. 38, Lspp. 46, Lspp. 48, Lspp. 49 were for AM only.

In conclusion, in this study the ethanolic extracts acted against Listeria spp. of equal or superior effectiveness that the antibiotics of clinical use analyzed. The use of uvilla extracts could be a viable alternative to act against pathogens, and could be part of naturally occurring antimicrobials.

\section{Acknowledgment}

The authors would like to thank al Departamento de Investigación de la Universidad Estatal de Bolívar for the financing of this work and his support provided.

\section{References}

Areiza, N., Maldonado, ML., Rojano, B. 2013. Extracto acuoso de uchuva (Physalis peruviana): actividades antiproliferativa, apoptótica y antioxidante. Perspectivas en Nutrición Humana. 15 (1): 41-45.

Bayas Morejón, IF., González, A., and Ferrús MA. 2017. Detection, Identification, and Antimicrobial Susceptibility of Arcobacter spp. Isolated from Shellfish in Spain. Foodborne Pathogens and Disease, ahead of print. doi:10.1089/fpd.2016.2202.

Çakir, Ö., Pekmez, M., Çepni, E., Candar, B., and Fidan, K. 2014. Evaluation of biological activities of Physalis peruviana ethanol extracts and expression of Bcl-2 genes in HeLa cells. Food Science and Technology (Campinas). 34(2): 422430. https://dx.doi.org/10.1590/fst.2014.0 060

Chavan, MD., Bansode, DS. 2015. Studies On Effect Of Guava Leaves Extract Against Selected Enteric Bacteria, Asian Journal of Pharmaceutical Technology and Innovation. 03 www.asianpharmtech.com.

Corrales-Bernal, A.,Vergara, AI., Rojano, B. Yahia, E., Maldonado, ME. 2015. Características nutricionales y antioxidantes de la uchuva colombiana (Physalys peruviana L.) en tres estadios de su maduración, Archivos Latinoamericanos de Nutrición. 65 (4): 254-262.

Coyle, MB. 2005. Manual of Antimicrobial Susceptibility Testing. American Society for Microbiology. 241 pages.

Dirección Nacional de Vigilancia Epidemiológica (DNVE-Ecuador). (2016). Listeriosis en el Ecuador. Gaceta epidemiológica 2016.

Göztok, F and Zengin, F. 2013. The antimicrobial activity of Physalis peruviana L. Bitlis Eren Univ Journal of Science and Technology. 3: 15-17.

Huang, KS., Yang, CH., Huang, SL., Chen, CY., Lu YY and Lin, YS. 2016. Recent Advances in Antimicrobial Polymers: A Mini-Review. International Journal of Molecular Sciences. 17: 1578; doi:10.3390/ijms17091578.

ISO 11290-1:1996. 1996. Microbiology of food and animal feeding stuffs e horizontal method for the detection and enumeration of Listeria monocytogenes e part 1: detection method. Geneva, Switzerland: International Standardisation Organisation (ISO).

Kushwaha, S., Betsy, A., and Chawla, P. 2012. Effect of Ashwagandha (Withania somnifera) Root Powder Supplementation in Treatment of Hyper tension. Ethno Med. 6:111-15.

Ministerio de Salud Pública del Ecuador (MSP). 2014. Reporte de enfermedades infecciosas durante el periodo 2013.

Murray, R. 2005. Manual of Clinical Microbiology, ASM Press, Washington, DC, USA, 6th edition.

Nair, R., Kalariya, K., Chanda, S. (2005). Antibacterial Activity of Some Selected Indian Medicinal Flora. Turkish Journal 
of Biology. 29: 41-47.

Otobone, FJ., Sanchez, AC., Nagae, R., Martins, JV., Sela, VR., De Mello, JC., Audi, EA. (2007). Effect of lyophilized extracts from guaraná seeds [Paullinia cupana var. sorbilis (Mart.) Ducke] on behavioral profiles in rats, Phytotherapy Research.21 (6): 531-536.

Ramadan, MM., El-Ghorab, AH., Ghanem, KZ. 2015. Volatile compounds, antioxidants, and anticancer activities of Cape gooseberry fruit (Physalis peruviana L.): An in-vitro study. Journal of the Arab Society for Medical Research. 10: 56-64.

Randazzo, W., Jiménez-Belenguer, A., Settanni, L., Perdones, A., Moschetti, M., Palazzolo, E., Guarrasi, V., Vargas, M., et al. 2015. Antilisterial effect of citrus essential oils and their performance in edible film formulations. Food Control. 59: 750-758.

Settani, L., Randazzo, W., Palazzolo, E., Moschetti, M., Aleo, A., Guarrasi, V., et al. (2014). Sensorial variations of antimicrobial activity and chemical composition of essential oils extracted from three Citrus limón L. Burm. cultivars. Natural Product Research. 28 (6): 383-391.

Shantha, S.M., and Gopal, S. 2014. Incidence of Listeria Species in Food and Food Processing Environment: A Review. Journal of Microbiology and Biotechnology. 3(1): 1-12.

Shokeen, P., Bala, M., Tandon, V. 2009.
Evaluation of the activity of 16 medicinal plants against Neisseria gonorrhoeae. International Journal of Antimicrobial Agents. 33(1): 86-91.

Suzgec-Selcuk, S., and Birteksoz, A.S. 2011. Flavonoids of Helichrysum chasmolycicum and its antioxidant and antimicrobial activities. South African Journal of Botany, 77: 170-174.

Thu Vu, T., Kim, H., Tran, VK., Le Dang, K., Nguyen, HT., Kim, H., Kim, IS., Choi, GI and Kim, JC. 2016. In vitro antibacterial activity of selected medicinal plants traditionally used in Vietnam against human pathogenic bacteria. Complementary and Alternative Medicine. 16:32, DOI 10.1186/s12906016-1007-2.

Yen, CY., Chiu, CC., Chang, FR., Chen, JY., Hwang, CC., Hseu, YC., Yang, HL., Lee, AY., Tsai, MT., Guo, ZL., Cheng, YS., Liu, YC., Lan, YH., Chang, YC., Ko, YC., Chang, HW., Wu YC. 2010. 4betaHydroxywithanolide E from Physalis peruviana (golden berry) inhibits growth of human lung cancer cells through DNA damage, apoptosis and G2/M arrest. BMC Cancer. 10: 46, doi: 10.1186/1471-240710-46.

Yilmaztekin, M. (2014). Analysis of volatile components of Cape gooseberry (Physalis peruviana L.) grown in Turkey by HSSPME and GC-MS. The Scientific World Journal. ID 796097, 8 pages.

\section{How to cite this article:}

Ma. Bernarda Ruilova Cueva, Rosa Angelica Tigre León, Ma. Magdalena López, Angel Yanchaliquín, Isidro Favian Bayas Morejón and Herminia Sanaguano Salguero. 2017. Antibacterial Effects of Uvilla (Physalis peruviana L.) Stracts againts Listeria spp. Isolated from Meat in Ecuador. Int.J.Curr.Microbiol.App.Sci. 6(4): 1146-1153.

doi: https://doi.org/10.20546/ijcmas.2017.604.141 